accelerated heavy hydrogen ions, we have noticed repeatedly the production of remarkably strong ionising radiations when silver was placed in the beam. Silver is close to tin in the periodic table and it is probable, therefore, that the effects observed by Dr. Ollano are due to the neutrons in the radiation from $\mathrm{Po}+\mathrm{Be}$, and that the phenomenon is general in elements in that part of the periodic table.

M. L. OLIPHANT.

Cavendish Laboratory, Cambridge. Oct. 27.

\section{Vision in the Ultra-Violet}

IN a letter in Nature of September 15, p. 416, Mr. Goodeve refers to a paper by Saidman and Dufestel ${ }^{1}$ on vision in the ultra-violet. Mr. Goodeve is probably unaware of a more recent paper by Saidman $^{2}$ on the same subject, where evidence is given of vision down to the mercury line 3130 . Furthermore, Saidman has pointed out another remarkable fact worthy of note; that is, that vision at short wave-lengths is possible only in young people; the limit of visibility recedes towards longer wave-lengths with increase in age.

I can support this statement from the case of my own eyes. Twenty-five years ago, I was able to see the 3650 mercury line very well (I did not try with shorter wave-lengths, on account of the difficulty of excluding diffuse light). At the present time, at the age of sixty-seven years, I cannot see the 3650 line at all. 'My sight, in other ways, has remained perfectly normal, except the inevitable far-sightedness and slight hypermetropia. I can see the $K$ line of calcium (3933) quite well.

This continuous retrogression in the limit of visibility is, without doubt, due to progressive absorption with age by the crystalline lens. As Saidman has remarked, the determination of the limit of visibility would give an indication of the age of the crystalline lens.

Nevertheless, certain precautions are necessary when determining this limit. It is necessary, in particular, to avoid all traces of diffuse light. Saidman used a mercury lamp with filters ; but the number of intense lines is not large enough to fix a precise limit. From this point of view, a richer spectrum, such as that of iron, or even a continuous spectrum would be better ; but there must be complete elimination of diffuse light. The use of a double spectroscope would probably be necessary in order to get a perfectly pure spectrum.

\section{University of Paris.}

Charles Fabry. Oct. 18.

${ }^{3}$ C.R., 182, 1173; 1926 ,

\section{Development of the Spark Discharge}

Ir has recently been shown ${ }^{1}$ that in the lightning discharge a preliminary discharge from cloud to ground occurs before the passage of the main stroke in the reverse direction. The luminous intensity of this preliminary discharge is much lower than that of the succeeding main stroke and its velocity of propagation is considerably smaller. The main stroke begins at the moment the preliminary leader reaches the ground and follows the leader path in the reverse direction.
Now it has been observed in the laboratory for sorne years that when an impulse voltage is applied to an asymmetrical gap (such as a point-plane gap) the voltage of the impulse being insufficient to cause spark-over, a discharge proceeds from the high voltage point electrode towards the plane electrode, the length of the discharge varying with the applied voltage. Under conditions favourable for observation, this discharge can be followed almost to the plane electrode when the voltage is just insufficient to cause spark-over. It is natural therefore to suppose that this preliminary discharge forms the conducting path for the main discharge when spark-over occurs, thus providing an exact analogue of the lightning discharge. The difficulties of observing this are connected with the short time available for the development of the preliminary discharge and its weak visual intensity.

To overcome these difficulties a camera was constructed consisting of a wide aperture lens and a
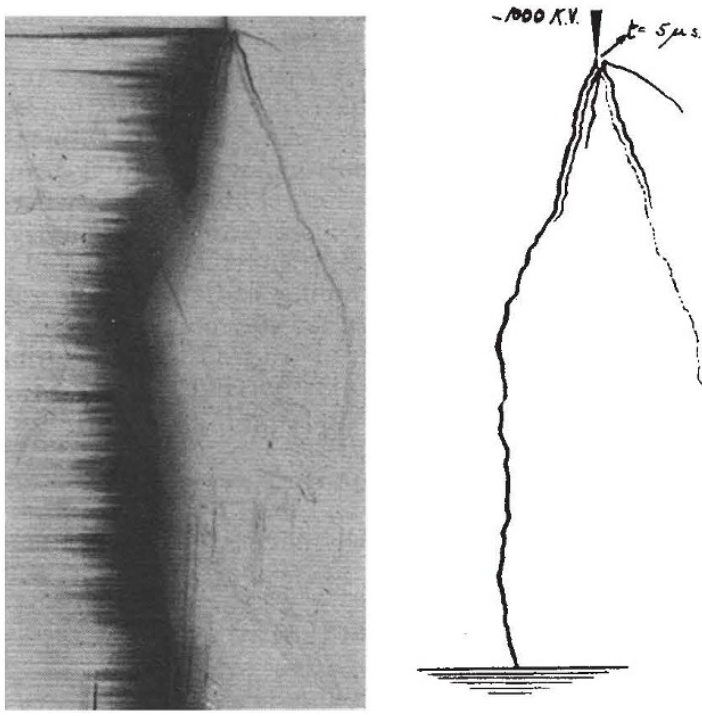

FIG. 1.

rotating film having a peripheral speed of 40 metres per second. With this resolving power, it has been possible to photograph the preliminary discharge well separated from the subsequent main discharge at voltages of the order of one to two million volts. The photographs show the course of the preliminary leader for something like 16-20 per cent of the electrode spacing, the luminosity falling off with distance from the initiating electrode. But over this distance the main discharge is seen to follow the path of the leader in all its details, and there is some evidence that where branching of the leader stroke occurs, the subsequent main stroke branches likewise. In Fig. 1 is shown on the left a record of the discharge between a negative point at $-1,000 \mathrm{k} . \mathrm{v}$. and a grounded plane, 40 inches apart; and on the right a sketch indicating the extent to which the leader can be followed in the original.

Evidence obtained from Boys's camera investigations indicates that the speed of the camera here used can give rise to no displacement of the image of the main discharge. We therefore interpret an observed diminution in the separation between leader and main discharges as arising from the time occupied. in the progress of the leader. 